\title{
Primary Diffuse Large B-Cell Lymphomas of the Temporoparietal Dura Mater and Scalp Without Intervening Skull Bone Invasion
}

\author{
-Case Report-
}

\author{
Hidenobu OCHIAI, Hirokazu KAWANO, Ryo MIYAOKA, \\ Noriaki KAWANO*, Yoshiya SHIMAO**, and Kensaku KAWASAKI*** \\ Departments of Neurosurgery, ${ }^{*}$ Hematology, and ${ }^{* *}$ Pathology, \\ Miyazaki Prefectural Hospital, Miyazaki; \\ *** Department of Neurosurgery, Kawasaki Neurosurgery Hospital, Miyazaki
}

\begin{abstract}
A 72-year-old man presented with an extremely rare case of primary diffuse large B-cell lymphomas (DLBCLs) of the dura and scalp existing independently without intervening cranial vault invasion. The patient presented with an indolent mass lesion at the left temporal parietal scalp. Magnetic resonance imaging and computed tomography revealed a solid homogeneously enhanced mass in the left temporoparietal scalp, and an extra-axial intracranial mass that existed just below the scalp without intervening skull invasion. The patient underwent gross total resection of these lesions via a left frontotemporoparietal craniotomy. Histological examination of the masses revealed DLBCLs. The patient received whole-brain radiation therapy, and subsequent chemotherapy with cyclophosphamide, adriamycin, vincristine, and prednisolone. He was discharged without neurological deficit. The present case of DLBCLs in the scalp and dura without intervening skull bone invasion indicates that malignant lymphoma should be considered in the differential diagnosis of scalp and dural tumors without intervening skull bone invasion.
\end{abstract}

Key words: B-cell lymphoma, dura, scalp, extranodal lymphoma, skull lymphoma

\section{Introduction}

Primary central nervous system lymphoma often arises from the midline or paraventricular regions of the brain. In contrast, primary extra-axial diffuse large B cell lymphoma (DLBCL) is rare, accounting for only $2.4 \%$ of the cases of primary central nervous system lymphoma, and usually occurs in the dura and/or cranial vault.8,9) The most common origin for primary malignant lymphoma of the dura mater is the cerebral convexity, followed by the falx, cerebellar tentorium, and suprasellar/parasellar regions. ${ }^{8,17)}$ These tumors often extend into the brain as well as into the skull and scalp, so sometimes appear as intracranial and extracranial tumors and often mimic meningiomas. ${ }^{1,17)}$ Primary DLBCL of the dura and scalp without intervening cranial vault invasion is extremely rare.

We describe a case of independently existing mass lesions of the scalp and dura in the left temporoparietal region that were identified as DLBCL.

Received June 16, 2009;

Accepted December 11, 2009

\section{Case Report}

A 72-year-old man with no previous medical history presented to another hospital with an indolent mass in the left temporoparietal region of the scalp. Computed tomography (CT) revealed mass lesions in the left temporoparietal subcutaneous region and an extra-axial mass just below the scalp mass without intervening skull bone invasion. The patient was subsequently referred to our hospital with a tentative diagnosis of subcutaneous and extradural hematomas.

On admission, an indolent, elastic hard mass, $7 \mathrm{~cm}$ in diameter, was observed in the left temporoparietal region (Fig. 1). Neurological examination revealed mild sensory aphasia. Skull radiography revealed no destruction or periosteal reaction of the skull beneath the subcutaneous region. Magnetic resonance (MR) imaging revealed a subcutaneous mass in the left temporoparietal region, which appeared hypointense on $\mathrm{T}_{1}$-weighted imaging, hyperintense on $\mathrm{T}_{2}$-weighted imaging, and homogeneously enhanced by gadolinium-diethylenetriamine penta-acetic acid (Gd-DTPA). MR imaging also revealed an intracranial extra-axial mass lesion in the left temporoparietal region with the same appearance as the scalp tumor. Dural tail 


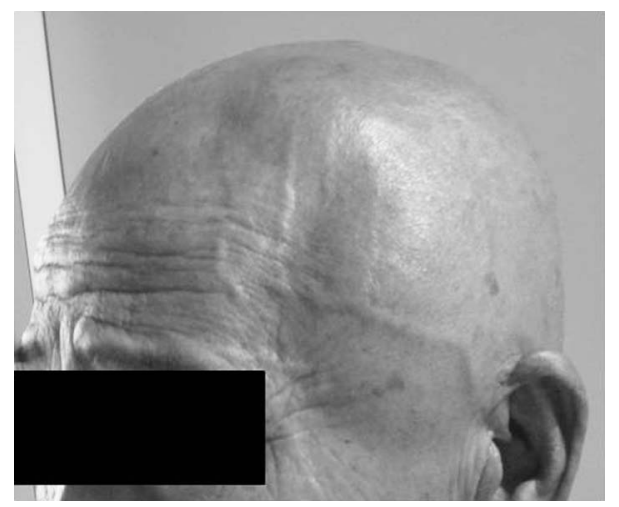

Fig. 1 Photograph showing a subcutaneous mass, about $7 \mathrm{~cm}$ in diameter, in the left temporoparietal region.
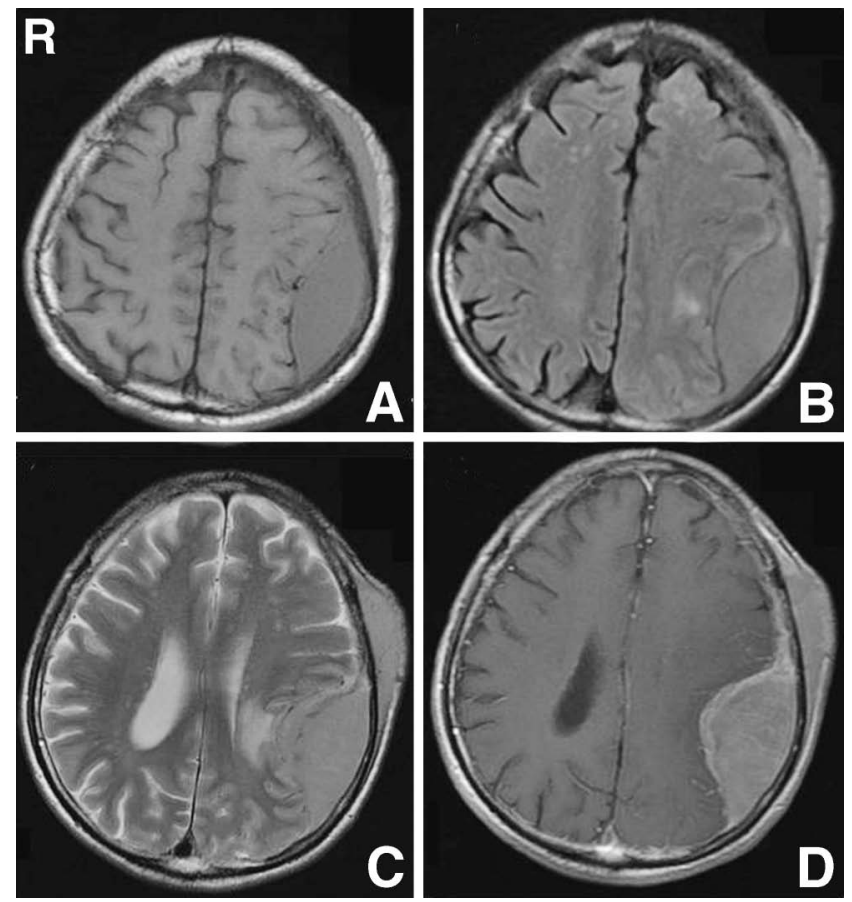

Fig. 2 Axial $\mathrm{T}_{\mathbf{1}}$-weighted (A), fluid-attenuated inversion recovery (B), $T_{2}$-weighted $(C)$, and $T_{1}$-weighted with contrast medium (D) magnetic resonance images showing a subcutaneous mass in the left temporoparietal region, which appeared hypointense on the $T_{1}$-weighted image, hyperintense on the $T_{2}$ weighted image, and homogeneously enhanced by gadoliniumdiethylenetriamine penta-acetic acid, and an intracranial extra-axial mass lesion in the left temporoparietal region, with dural tail sign. No intervening skull bone invasion of the tumor was observed.

sign was also observed for this tumor (Fig. 2). However, these mass lesions were thought to exist independently, as the preoperative neuroimaging findings did not reveal tumor invasion through the intervening skull bone (Fig. 3). Since MR imaging demonstrated multiple mass lesions, a metastatic tumor was suspected, so systemic chest and ab-
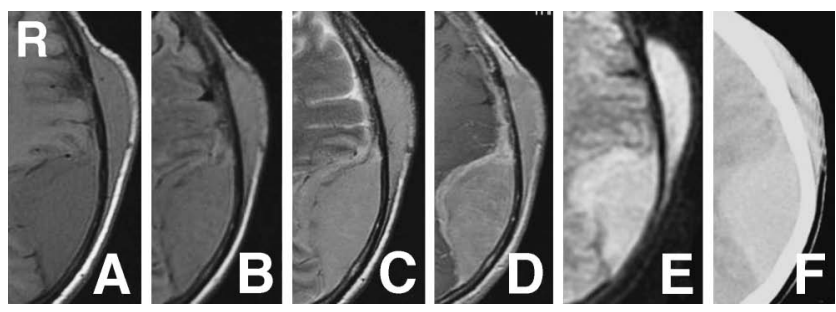

Fig. 3 Axial $\mathrm{T}_{1}$-weighted (A), fluid-attenuated inversion recovery (B), $T_{2}$-weighted $(C), T_{1}$-weighted with contrast medium (D), and diffusion-weighted (E) magnetic resonance images, and computed tomography scan $(F)$ showing no tumor invasion of the intervening bone.
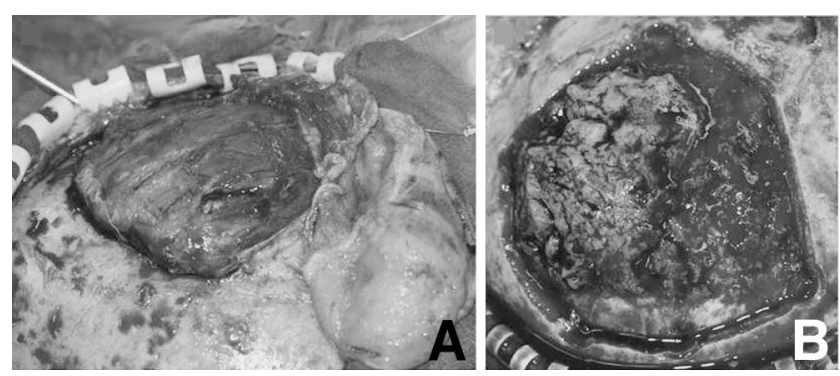

Fig. 4 Intraoperative photographs of the scalp tumor (A) and the extradural tumor (B).

dominal CT, positron emission tomography, and bone scintigraphy were performed. However, no other tumor lesion was detected. On the basis of these findings, the differential diagnoses were malignant lymphoma, metastatic tumors of unknown origin, and meningioma with some type of scalp tumor. The patient underwent a left temporoparietal craniotomy. Reflection of the scalp exposed a gray, elastic hard, and hypovascular tumor (Fig. 4A). This tumor did not invade the galea aponeurotica, but extended to the temporalis muscle and periosteum without invading the outer table of the skull. The tumor was eventually excised en-bloc, and a temporoparietal craniotomy was performed. Removal of the bone flap exposed a tumor that appeared identical to the scalp tumor, but no sign of invasion of the inner table of the skull. The tumor was mainly localized in the extradural space, but had partially destroyed the dura mater and invaded the pia mater over the left temporal lobe (Fig. 4B). Gross total resection of this mass was performed. The intraoperative findings confirmed that the tumors had not invaded the intervening skull, so the bone flap was returned to its original position. Histological examination of the specimens revealed diffuse proliferation of lymphoma cells which were positive for CD20 and CD79a, and negative for CD3 and CD45R0 (Fig. 5). The histological diagnosis was DLBCL.

The patient's sensory aphasia improved immediately after the operation. Postoperative bone CT confirmed absence of tumor invasion into the intervening skull (Fig. 6). Since the histological diagnosis of the tumors was DLBCL, 
the patient received whole-brain radiation therapy, and subsequent chemotherapy with cyclophosphamide, adriamycin, vincristine, and prednisolone (CHOP). He
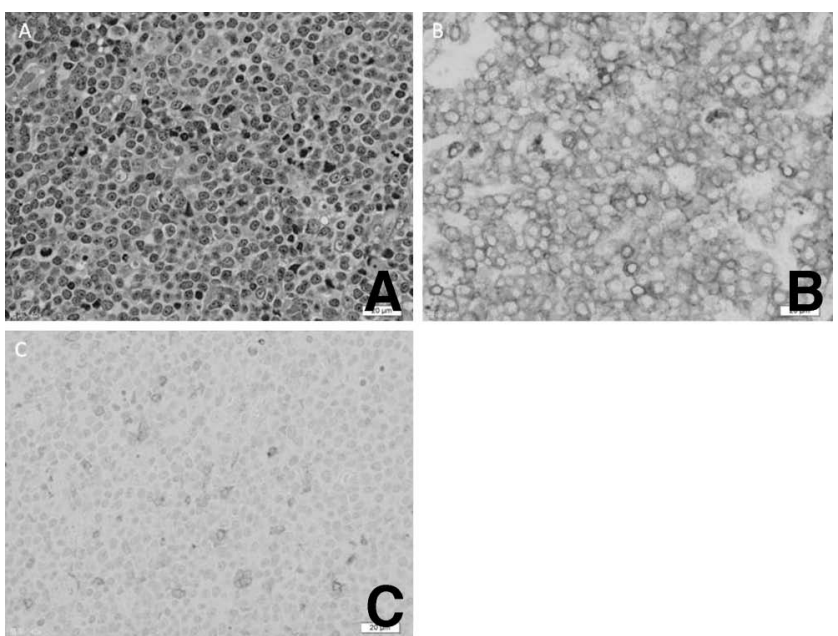

Fig. 5 Photomicrographs of the surgical specimen showing diffuse proliferation of lymphoma cells (A), which were positive for CD20 and CD79a (B), and negative for CD3 and CD45RO (C). A: hematoxylin-eosin stain, B: immunohistochemical staining for CD79a, C: immunohistochemical staining for CD3, original magnification $\times 200$.

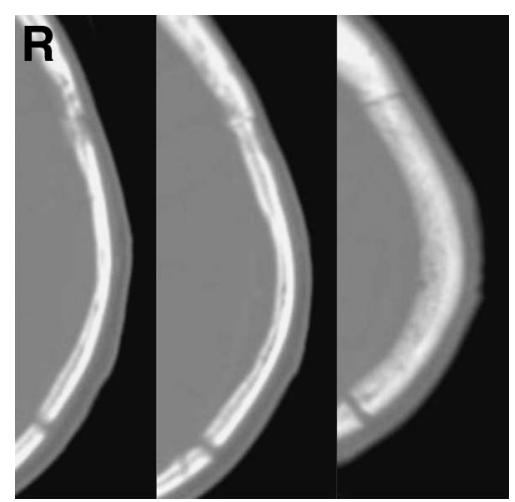

Fig. 6 Postoperative bone image computed tomography scans showing no tumor invasion into the intervening skull. was discharged without neurological deficit.

\section{Discussion}

Dural or cranial vault malignant lymphomas are generally associated with extra- and intracranial invasion, and such cases always extend from the intracranial to the extracranial space with intervening skull bone destruction. ${ }^{2-6,10,11,13-15,18,19)}$ In contrast, in our case, the dural and subcutaneous tumors existed independently without intervening skull invasion as confirmed by the preoperative images and surgical findings. Histological diagnosis confirmed these tumors as DLBCLs. The exact mechanisms underlying the independent existence of these tumors and their extension into the outside and inside of the skull remain unknown. However, two mechanisms can be considered: the tumor first arose in the dura or scalp and then extended intracranially or extracranially through the emissary vein without invading the skull ${ }^{3,7}$; and the multiple tumors are attributable to the multi-centric nature of the malignant lymphoma. In our case, the emissary vein of the skull between the intracranial and extracranial tumor was not well developed, so the second mechanism seems most likely.

The differential diagnosis of tumors that arise and extend both intracranially and extracranially includes primary skull tumors, metastatic skull tumors, and intraosseous meningiomas. ${ }^{13)}$ However, these tumors often invade and destroy the intervening skull bone. Only 3 cases of scalp and intracranial lymphoma without signs of intervening skull bone destruction have been reported (Table 1)..$^{7,12,16)}$ The final diagnoses in these cases were undifferentiated large cell lymphoma, Hodgkin's disease, and DLBCL. ${ }^{7,12,16)}$ The tumors in 2 cases arose in the parietal region and the other tumor arose in the frontal region. ${ }^{7,12,16)}$ Therefore, the presence of tumors in both the scalp and dura without intervening skull bone invasion might be one of the characteristics of malignant lymphomas. Another diagnostic clue for scalp and dural tumors that do not invade the intervening skull bone might be the differentiation of the dural tumor from meningioma, since both types have many neuroradiological features: these extra-axial tumors, appear as hypointense or isointense on MR imaging, with homogeneous enhancement and dural tail sign after administration of Gd-DTPA.1,8,17) However, underlying vasogenic edema appears to be more common

Table 1 Summary of the clinical features of patients with primary diffuse large B-cell lymphoma (DLBCL) of the dura mater and scalp without intervening skull bone invasion

\begin{tabular}{|c|c|c|c|c|c|c|}
\hline Author (Year) & $\begin{array}{l}\text { Age (yrs)/ } \\
\text { Sex }\end{array}$ & Location & Surgery & Histology & $\begin{array}{l}\text { Adjuvant } \\
\text { treatment }\end{array}$ & Outcome \\
\hline Tomaszek et al. (1984) ${ }^{16)}$ & $23 / \mathrm{M}$ & rt parietal & biopsy & Hodgkin's disease & none & dead (2 yrs) \\
\hline Holtas et al. $(1985)^{7)}$ & $60 / \mathrm{F}$ & lt frontal & biopsy & $\begin{array}{l}\text { undifferentiated } \\
\text { large cell } \\
\text { lymphoma }\end{array}$ & steroid & alive (6 mos) \\
\hline Nishimoto et al. $(2003)^{12)}$ & $63 / \mathrm{M}$ & lt parietal & biopsy & DLBCL & CHOP & alive \\
\hline Present case & $72 / \mathrm{M}$ & lt temporoparietal & total resection & DLBCL & WBRT + CHOP & alive (1 yr) \\
\hline
\end{tabular}

CHOP: cyclophosphamide, adriamycin, vincristine, and prednisolone; WBRT: whole brain radiation therapy. 
in cases of primary dural lymphoma. ${ }^{17)}$ If a dural tumor shows these features and the probable diagnosis is dural lymphoma, then the scalp tumor could also be lymphoma.

Accurate histological diagnosis of the tumor is indispensable to establish the appropriate treatment. The histological diagnosis of a scalp tumor can be easily achieved by needle biopsy. However, we did not perform needle biopsy for the scalp tumor and excised both scalp and intracranial tumors, because we were unable to validate the common origin of the intracranial and scalp tumors. We also resected the intracranial tumor since it was considerably large and symptomatic. A previous case of 63-year-old man with a frontoparietal mass and an underlying very small dural lesion was diagnosed on the basis of the findings of the scalp tumor biopsy. ${ }^{12)}$ The dural lesion was diagnosed by its response to CHOP therapy. ${ }^{12)}$ Therefore, we think that if the intracranial tumor is small and the scalp tumor is diagnosed as a lymphoma on the basis of the biopsy findings, radiation therapy or chemotherapy prior to resection of the intracranial tumor, and monitoring the response to these treatments would be a good treatment strategy, since tumors located within the scalp and dura without intervening skull invasion may indicate malignant lymphomas.

The present case of DLBCLs in the scalp and dura without intervening skull bone invasion is extremely rare, but indicates that malignant lymphoma should be considered in the differential diagnosis of scalp and dural tumors without intervening skull bone invasion.

\section{References}

1) Abgi CB, Bannister CM, Turnbull IW: Primary cranial vault lymphoma mimicking a meningioma. Neurochirurgia (Stuttg) 26: 130-132, 1983

2) Bhatia S, Smally AJ, Dekker P: Primary non-Hodgkin's lymphoma of the cranial vault. Clin Oncol (R Coll Radiol) 9: 195-196, 1997

3) Curty B, Kernan J, Favre J: Malignant non-Hodgkin's lymphoma of the cranial vault: a case report. Br J Neurosurg 11: 433-436, 1997

4) Freudenstein D, Bornemann A, Ernemann U, Boldt R, Duffer F: Intracranial malignant B-cell lymphoma of the dura. Clin Neuropathol 19: 34-37, 2000

5) Fukushima Y, Oka H, Utsuki S, Nakahara K, Fujii K: Primary malignant lymphoma of the cranial vault. Acta Neurochir (Wien) 149: 601-604, 2007

6) Herkes GK, Partington MD, O’Neill BP: Neurological features of cranial vault lymphomas: report of two cases. Neurosurgery 29: 898-901, 1991
7) Holtas S, Monajati A, Utz R: Computed tomography of malignant lymphoma involving the skull. J Comput Assist Tomogr 9: 725-727, 1985

8) Iwamoto FM, Abrey LE: Primary dural lymphomas: a review. Neurosurg Focus 21(5): E5, 2006

9) Iwamoto FM, DeAngelis LM, Abrey LE: Primary dural lymphomas: a clinicopathologic study of treatment and outcome in eight patients. Neurology 66: 1763-1765, 2006

10) Landys KE, Berg GEB, son Torgerson JS, Klingenstierna HAG, Ridell BS, Johansson BR: Bulky centroblastic nonHodgkin's lymphoma of the cranium vault mimicking brain involvement managed with chemotherapy. Cancer 76: 1261-1267, 1995

11) Morioka T, Tashima $T$, Nishio $S$, Nishie E, Fukui $M$, Okamura T: Malignant lymphoma of the scalp at the site of a previous blunt trauma: report of two cases. Surg Neurol 42: 117-120, 1994

12) Nishimoto $T$, Yuki K, Sasaki T, Imada $Y$, Murakami $T$, Kodama Y: [A case of subcutaneous malignant lymphoma with dura mater lesion]. No Shinkei Geka 31: 43-47, 2003 (Japanese)

13) Paige M, Bernstein JR: Transcalvarial primary lymphoma of bone. A report of two cases. Neuroradiology 37: 456-458, 1995

14) Pardhanani G, Ashkan K, Mendoza N: Primary non-Hodgkin's lymphoma of the cranial vault presenting with unilateral proptosis. Acta Neurochir (Wien) 142: 597-598, 2000

15) Sato $M$, Saito $S$, Yamaguchi K: [Primary malignant lymphoma of the skull presenting a huge mass lesion: case report]. No Shinkei Geka 21: 1061-1064, 1993 (Japanese)

16) Tomaszek DE, Tyson GW, Stang P, Bouldin T: Contiguous scalp, skull, and epidural Hodgkin's disease. Surg Neurol 21: 182-184, 1984

17) Tu PH, Giannini C, Judkins AR, Schwalb JM, Burack R, O'Neil BP, Yachnis AT, Burger PC, Scheithauer BW, Perry A: Clinicopathologic and genetic profile of intracranial marginal zone lymphoma: a primary low-grade CNS lymphoma that mimics meningioma. J Clin Oncol 23: 5718-5727, 2005

18) Villela LM, Blanco-Salazar A, Caballero R, Borbolla-Escoboza R: Aggressive lymphoma involving intracranial epidural region. J Neurooncol 83: 181-182, 2007

19) Yuki K, Kodama Y, Onda J, Emoto K, Kirimoto K, Yamane T, Sasaki N, Nanba K, Kamada N: Metastatic malignant lymphoma of the skull with extradural and extracranial extension. Case report. Neurol Med Chir (Tokyo) 29: 762-766, 1989

Address reprint requests to: Hidenobu Ochiai, M.D., Ph.D., Department of Neurosurgery, Miyazaki Prefectural Hospital, 5-30 Kitatakamatsu-cho, Miyazaki, Miyazaki 880-8510, Japan. e-mail: hochiai@fc.miyazaki-u.ac.jp 